\title{
DISTRIBUTED CONVEX OPTIMIZATION WITH LIMITED COMMUNICATIONS
}

\author{
Milind Rao ${ }^{\dagger} \quad$ Stefano Rini ${ }^{\star} \quad$ Andrea Goldsmith ${ }^{\dagger}$ \\ ${ }^{\dagger}$ Electrical Engineering, Stanford University, Stanford, CA \\ * National Chiao Tung University, Taiwan \\ $\{$ milind, andreag\}estanford.edu, stefano@nctu.edu.tw
}

\begin{abstract}
In this paper, a distributed convex optimization algorithm, termed distributed coordinate dual averaging (DCDA) algorithm, is proposed. The DCDA algorithm addresses the scenario of a large distributed optimization problem with limited communication among nodes in the network. Currently known distributed subgradient methods, such as the distributed dual averaging or the distributed alternating direction method of multipliers algorithms, assume that nodes can exchange messages of large cardinality. Such network communication capabilities are not valid in many scenarios of practical relevance. In the DCDA algorithm, on the other hand, communication of each coordinate of the optimization variable is restricted over time. For the proposed algorithm, we bound the rate of convergence under different communication protocols and network architectures. We also consider the extensions to the case of imperfect gradient knowledge and the case in which transmitted messages are corrupted by additive noise or are quantized. Relevant numerical simulations are also provided.
\end{abstract}

Index Terms - Distributed optimization, subgradient methods, convex analysis, wireless communications

\section{INTRODUCTION}

With the emergence in recent years of big data paradigms, decentralized optimization algorithms have received considerables interest in the literature. A distributed optimization problem of particular relevance is the one in which the global objective function is obtained as the sum of a local convex functions. Originally considered by Tsitsiklis et al. [1], this problem is broadly referred to as the consensus problem. A number of distributed subgradient methods have been proposed to solve the consensus problem such as the distributed dual averaging and the distributed alternating direction method of multipliers algorithms. A distributed subgradient (DSG) algorithm for the consensus problem is initially proposed in [2], building upon consensus algorithms for computing the exact averages of initial values at the agents [3]. In the algorithm of [2], each node updates its estimate using a linear combination of the estimates of its neighbors and the gradient of its local function. In the literature, a number of variations of this algorithm have been considered, such as continuous time extensions [4], networks with link failures [5], and quantized communication [6]. Another interesting variation of the DSG algorithm of [2] is the coordinate descent method in which, in order to reduce the dimension of the messages sent across a network, only one coordinate of the optimal solution is communicated at each time instant. For this problem, Liu et al [7] analyze an asynchronous distributed coordinate descent algorithm. Inspired by Nesterov's dual averaging algorithm [8], Duchi et al. 91 prose the distributed dual averaging (DDA) algorithm for the consensus problem. In this algorithm, each node maintains an estimate for a dual variable by averaging the estimates of its neighbors and adding the gradient. A proximal projection of the dual variable produces optimization variable. The dual variable is updated similarly to the DSG algorithm, while the dual projection allows to incorporate nonlinear constraints on the solution. In [9], the authors also study the performance of the DDA algorithm in the presence of time varying networks, communication of gossip protocols, and stochastic gradients. The analysis of the DDA algorithm with delays in the communication network is performed in [10 11]. The authors of [12] study the computation/communication trade-off for the DDA algorithm by considering the case in which communication is subject to a total cost constraint.

Another popular class of algorithms to solve distributed constrained convex optimization problems are distributed alternating direction method of multipliers (DADMM) algorithms. This class of algorithms was originally proposed in [13], building upon the ADMM algorithm of [14]. The analysis of convergence for this algorithm is performed in [15], while the case of asynchronous communications is studied in [16].

Contributions: Our main contribution is a decentralized distributed protocol that places rate constraints on the communication between nodes, and yet guarantees convergence to the optimal value at all nodes. This algorithm is inspired by the DDA algorithm of [9] and is thus termed the distributed coordinate dual averaging (DCDA) algorithm. In the following, we derive the convergence of DCDA algorithm for different communication protocols and network architectures. Additionally, we study the behavior of the algorithm in the scenario of a stochastic gradient, noisy and quantized communication.

\section{PROBLEM FORMULATION}

We study the distributed optimization problem in which the minimum of a function is to be computed when factors of this functions are distributed across a network subject to communication constraints. Consider the $n$-nodes undirected graph $G=(V, E), V=[1: n]$, and $E \subset V \times V$ in which each the node $V_{i}$ is associated the function $f_{i}: \mathbb{R}^{d} \rightarrow \mathbb{R}$. Each function $f_{i}$ is a factor of the linear combination

$$
f(x)=\sum_{i=1}^{n} f_{i}(x),
$$

for $x \in \mathcal{X}$ with $\mathcal{X}$ closed and convex. We assume that each $f_{i}(x)$ is convex and L-Lipschitz with respect to a norm $\|\cdot\|$ or $\left|f_{i}(x)-f_{i}(y)\right| \leq L\|x-y\|, \quad x, y \in \mathcal{X}$. The Lipschitz condition implies that for any $x \in \mathcal{X}$ and any subgradient $g_{i} \in$ $\partial f_{i}(x)$, we have $\left\|g_{i}\right\|_{*} \leq L$. At each time instant $t \in \mathbb{N}$, the node $V_{i}$ maintains an estimate $x_{i}(t)$ of the value $x^{*}$ which attains the minimum of the function $F(x)$ in (1). The node $V_{i}$ is able to communicate to the node $V_{j}$ at the time instant 
$t$ if the two nodes are connected by an edge $E$ in $G$. Let $A$ be the symmetric incidence matrix of $G$ प Some examples of a network are:

- fully-connected network: in which $A=11^{\top}-\mathrm{I}$,

- random network: in which two nodes are connected with probability $p$,

- ring network: in which $A_{i j, i \neq j}=1$ iff $|i-j| \bmod n \leq l$ for some $l \in \mathbb{N}$. In this configuration, nodes are arranged in a circle and a node is connected to $l$ neighbors on either sides.

Upon receiving a message from its neighboring nodes, each node $V_{i}$ updates its estimate of the minimum value, $x_{i}(t)$. In the distributed optimization problem, the goal is to determine a set of communication strategies and estimate update rules such that each $x_{i}$ converges to $x^{*}$ as time grows to infinity.

In the following, given the time sequence $c(t) \in \mathbb{R}^{n}$, we will denote the time and space average as $\widehat{c}(t)$ and $\bar{c}(t)$ respectively, i.e.

$$
\widehat{c}_{i}(t)=\frac{1}{t} \sum_{t^{\prime}=1}^{t} x_{i}\left(t^{\prime}\right), \quad \bar{c}(t)=\frac{1}{n} \sum_{i=1}^{n} c_{i}(t) .
$$

The DCDA algorithm: In the DCDA algorithm, each node $V_{i}$ maintains both an estimate of the optimization variable, $x_{i}(t)$, and its dual variable, $z_{i}(t)$. At each time instant, both the primal variable and the dual variables are updated according to the message received from the neighboring nodes and the subdifferential of the objective function $f_{i}$ in the primal estimate $x_{i}, g_{i}(t)$. More precisely, the communication and update rules are as follows. At each iteration, each node $i$ broadcasts a subset of its $d$ coordinates of the dual variable $z(t)$ to a subset of of its neighbors. For instance, node $i$ broadcasts coordinate $k$ to neighbors $N^{k}(i)$. The update of the dual variable is a component wise update

$$
\left[z_{i}(t+1)\right]_{k}=\sum_{j \in N^{k}(i)} P_{i j}^{k}(t)\left[z_{j}(t)\right]_{k}+\left[g_{i}(t)\right]_{k} \quad \forall k,
$$

where $P^{k}(t)$ is a doubly stochastic matrix and where $P_{i j}^{k}>0$ if and only if $A_{i j}>0$ and the node $j$ is broadcasting the set of coordinates $k$ to node $i$. In the following, we consider three different policies for the selection of the coordinate $k$ broadcasted by the nodes:

- static sharing scheme: at each time instant, nodes transmit the same coordinates to their neighbors, corresponding to $P^{k}(t)=P^{k}$ for some fixed $P^{k}$.

- round robin scheme: in which the $k^{\text {th }}$ coordinate is shared every $\pi$ time instances, corresponding to $P^{k}(t)=P_{\pi}$ when $t=n \pi+k$ for some $n \in \mathbb{N}$, else $P^{k}(t)=\mathbf{I}$.

- randomized scheme: in which nodes randomly and uniformly select the coordinate to be transmitted in each time instant.

Note that the stated sharing scheme with $P^{k}=P$ corresponds to the DDA of [9]: this corresponds to the case when nodes broadcast their entire dual variable to their neighbors. Also note that, given a symmetric adjacency network $A^{k}(t)$ for coordinate $k$ at time $t$, we can obtain the doubly stochastic matrix $P^{k}(t)$ as

$$
D^{k}(t)=\operatorname{diag}\left(A^{k}(t) \mathbf{1}\right), \quad P^{k}(t)=\mathbf{I}-\frac{D^{k}(t)-A^{k}(t)}{\max _{i} D^{k}(t)_{i i}+1} .
$$

${ }^{1}$ That is, $A_{i j}$ is non-zero only if nodes $i$ and $j$ are neighbors.
At each time instant, the primal variable $x_{i}(t+1)$ is computed from $z_{i}(t+1)$ as:

$$
x_{i}(t+1)=\Pi_{\psi, \alpha(t)}\left(z_{i}(t+1)\right)
$$

The function $\Pi_{\psi, \alpha(t)}$ is type of non-linear proximal projection and is used to stabilize estimates of the primal variable and ensure that optimization constraints are satisfied. It is defined as

$$
\Pi_{\psi, \alpha(t)}\left(z_{i}(t)\right)=\operatorname{argmin}_{x}\left\langle x, z_{i}(t)\right\rangle+\frac{1}{\alpha(t)} \psi(x) .
$$

the $\{\alpha(t)\}_{t=0}^{\infty}$ is a non-increasing sequence of positive stepsizes which typically scales as $1 / \sqrt{t}$. Also, $\psi: \mathbb{R}^{d} \rightarrow \mathbb{R}$ is a proximal function, that is assumed to be 1 -strongly convex with respect to norm $\|\cdot\|$. and positive defined. Examples of a proximal function include:

- squared proximal function: $\psi(x)=\frac{1}{2}\|x\|_{2}^{2}$ is 1-strongly convex with respect to the $\ell_{2}$-norm.

- entropic proximal function: $\psi(x)=\sum_{k=1}^{d} x_{i} \log x_{i}-x_{i}$ is 1 -strongly convex with respect to the $\ell_{1}$-norm.

The performance of the DCDA algorithm is studied in terms of the convergence to zero of the term $f\left(\hat{x}_{i}(T)\right)-f\left(x^{*}\right)$. rithm:

Finally, we consider three extensions of the DCDA algo-

- stochastic gradient: in which the objective function subgradient is not exactly known at each node,

- noisy communication: in which transmissions are corrupted by additive noise,

- quantized communications: in which transmissions are quantized before communication.

\section{MAIN RESULTS}

The main results of the paper consists of the characterization of the DCDA convergence rate for different coordinate selection policies and communication networks.

Theorem 1. Let the sequences $\left\{x_{i}(t)\right\}_{t=0}^{\infty}$ and $\left\{z_{i}(t)\right\}_{t=0}^{\infty}$ be generated by the updates (3) and (2) with step size sequence $\{\alpha(t)\}_{t=0}^{\infty}$. Then for any $x^{*} \in \mathcal{X}$ and for each node $i \in V$, the DCDA algorithm is such that

$f\left(\hat{x}_{i}(T)\right)-f\left(x^{*}\right) \leq \frac{\psi\left(x^{*}\right)}{T \alpha(t)}+\frac{1}{T} \sum_{t=1}^{T} \alpha(t-1)\|\bar{g}(t)\|_{*}^{2}$
$+\frac{2 L}{n T} \sum_{t=1}^{T} \sum_{j=1}^{n} \alpha(t)\left\|\bar{z}(t)-z_{j}\right\|_{*}+\frac{L}{T} \sum_{t=1}^{T} \alpha(t)\left\|\bar{z}(t)-z_{j}\right\|_{*}$.

The result in Th. 1 is substantially equivalent to the result, of [9. Th. 1]. The first two terms in (5) are optimization error terms common to sub-gradient algorithms while the last two are penalties incurred due to having different estimates at different nodes in the network or the penalty from consensus. The result in Th. 1 can be further developed for specific communication protocols.

Lemma 2. Static sharing scheme: For the settings in Th. 1. the DCDA algorithm under the static coordinate sharing scheme is such that

$$
\begin{aligned}
& f\left(\hat{x}_{i}(T)\right)-f\left(x^{*}\right) \leq \frac{\psi\left(x^{*}\right)}{T \alpha(T)} \\
& +\frac{L^{2}}{T} \sum_{t=1}^{T} 4 \alpha(t-1)\left(\frac{2 \min (d, n) \log \sqrt{n} d T}{1-\max _{k} \sigma_{2}\left(P^{k}\right)}+3\right) .
\end{aligned}
$$

where $\sigma_{2}(M)$ is the second largest eigenvalue of $M$. 
Lem. 2]implies that for the choice of $\alpha(t)=C / \sqrt{t}$ for an appropriate $\mathrm{C}$, the error scales as $L \sqrt{\frac{\min (d, n) \log \left(n^{1 / 2} d T\right)}{T\left(1-\max _{k} \sigma_{2}\left(P^{k}\right)\right)}}$. The error scales as $T^{-1 / 2}$ which is a common factor we see in all results. The term $1 / 1-\sigma_{2}\left(P^{k}\right)$ determines how quickly nodes come to a consensus in coordinate $k$. When $P^{k}=P$, we do not obtain the factor $\min (d, n)$ retrieving the results of DDA [9].

Lemma 3. Round robin scheme: For the settings in Th. 1 the $D C D A$ algorithm under the round robin $m$-coordinate sharing scheme is such that

$$
\begin{aligned}
& f\left(\hat{x}_{i}(T)\right)-f\left(x^{*}\right) \leq \frac{\psi\left(x^{*}\right)}{T \alpha(T)} \\
& +\frac{L^{2}}{T} \sum_{t=1}^{T} \alpha(t-1)\left(10+\frac{12 d \log 2 \sqrt{n} T}{m\left(1-\sigma_{2}(P)\right)}\right) .
\end{aligned}
$$

With an appropriate choice of the step size, the error in Lem. 3 scales as $L \sqrt{\frac{d \log (n T)}{m T\left(1-\sigma_{2}(P)\right)}}$. Thus, we would need twice the amount of time to achieve a fixed error $\epsilon$ if we transmit half the number of coordinates $m$ at each time instant.

Lemma 4. Randomized scheme: For the settings in Th. 1 the DCDA algorithm under the randomized coordinate sharing scheme is such that, with probability greater than $1-\delta$

$$
\begin{aligned}
& f\left(\hat{x}_{i}(T)\right)-f\left(x^{*}\right) \leq \frac{\psi\left(x^{*}\right)}{T \alpha(T)} \\
& +\frac{L^{2}}{T} \sum_{t=1}^{T} \alpha(t-1)\left(10+18 \frac{\min (d, n) \log T d n^{1 / 3} / \delta}{1-\max _{k} \sigma_{2}\left(\mathbb{E}\left[P^{k}(t)^{2}\right]\right)}\right)
\end{aligned}
$$

The result in Lem. 4 is similar to the static coordinate sharing case with the expected doubly stochastic sharing matrix used. Consider the specific case where the nodes collectively share coordinate $k$ with all other nodes with probability $\rho$. In this case, $P^{k}(t)=\frac{1}{n} \mathbf{1 1}{ }^{\top}$ with probability $\rho$, else $P^{k}(t)=\mathbf{I}$. In this case, the error scales as $L \sqrt{\frac{\log T d n / \delta}{\rho T}}$ with high probability. Similar to the round robin case, the analysis shows an inverse dependence between the number of coordinates shared and the time needed for convergence.

\subsection{Variations of the DCDA algorithms}

In this section we study three variations of the DCDA scheme as introduced in Sec. 2 First, we consider the case in which each node does not have access to the exact gradient of its local function but instead obtains a noisy estimate of this value. The DCDA algorithm for the stochastic gradient setting simply uses the stochastic gradient in place of the actual gradient. Convergence is studied under some mild assumptions on the noisy gradient value.

Assumption 1. Assume $\mathcal{F}_{t}$ be the $\sigma$-field that contains all information known by all nodes till time $t$ and let $g^{\prime}(t)$ be the stochastic gradient at time $t$. Further assume that:

- the stochastic gradient $g_{i}^{\prime}(t)$ is an unbiased estimate of the actual gradient, i.e. $\mathbb{E}\left[g_{i}^{\prime}(t) \mid \mathcal{F}_{t}\right] \in \partial f_{i}\left(x_{i}(t)\right)$,

- the stochastic gradient is bounded. $\left\|g_{i}^{\prime}(t)\right\|_{*} \leq L$

- The set $\mathcal{X}$ satisfies $\left\|x-x^{\prime}\right\| \leq R \forall x, x^{\prime} \in \mathcal{X}$.
Lemma 5. Stochastic gradient DCDA algorithm: For the settings in Th. 1 and under the assumptions in Ass. 11 the stochastic gradient DCDA algorithm is such that, with probability $1-\delta$

$$
\left.f\left(\hat{x}_{i}(T)\right)-f\left(x^{*}\right) \leq \sqrt{5}\right)+L R \sqrt{\frac{8 \log \frac{1}{\delta}}{T}} .
$$

From Lem. 5 we conclude that the scaling of the error of the stochastic gradient DCDA algorithm is the same as the DCDA algorithm.

Let us next consider the noisy communication scenario. More precisely, message $z_{j}(t)$ transmitted at time $t$ from node $j$ to node $i$ suffers from additive noise $n_{i j}(t)$, i.e. $u_{i j}(t)=$ $z_{j}(t)+n_{i j}(t)$

The DCDA algorithm for the noisy communication setting uses the noisy dual variable estimate $u_{i j}(t)$ instead of the actual value $z_{j}(t)$. Convergence is shown under some assumptions on the noise sequence and for the static sharing scheme.

Lemma 6. Noisy communication static staring scheme DCDA algorithm: Consider the settings in Th. 1 scheme where the function is L-Lipschitz, with respect to the $\ell_{2}$-norm. Further assume that there exists $R$ such that $\sup _{x, x^{\prime} \in \mathcal{X}} \| x-$ $x^{\prime} \|_{2} \leq R$. Under the assumptions that each $n_{i j}(t)$ has independent zero-mean sub-Gaussian components of power $\gamma^{2} / d$, the noisy communication static sharing DCDA algorithm is such that, with probability $1-\delta$

$$
\begin{aligned}
& f\left(\hat{x}_{i}(T)\right)-f\left(x^{*}\right) \leq 6+\gamma(R+2 L) \sqrt{\frac{2 \log \frac{3}{\delta}}{n T}}+\sum_{t=1}^{T} \alpha(t-1) \\
& \times\left(\frac{\gamma^{2}\left(1+\sqrt{8} \log \frac{3}{\delta}\right)}{n d T}+\frac{3 L}{T} \sqrt{\frac{2 \gamma^{2} \log \frac{6 T n d}{\delta}}{\left(1-\max _{k} \sigma_{2}\left(P^{k}\right)^{2}\right)}}\right)
\end{aligned}
$$

Finally, we consider the case in which the communication among nodes is quantized using infinite-level uniform quantization. At each time step, a node broadcasts the quantized scaled dual variable update

$$
\left[u_{i}(t)\right]_{k}=\left\lfloor\frac{\left[z_{i}(t)\right]_{k}-\left[z_{i}(t-1)\right]_{k}}{s(t)}+u(t)\right\rfloor,
$$

where $u(t) \stackrel{\text { iid }}{\sim} \mathcal{U}([-1 / 2,+1 / 2])$ is dither used to guarantee that the quantization noise is uniformly distributed in the interval $[-1 / 2,+1 / 2]$, while $s(t)>0$ is a zooming sequence that converges to zero and is known a priori to all nodes. The dual update operation is replaced by:

$\left[z_{i}(t+1)\right]_{k}=\left[z_{i}(t)+g_{i}(t)-g_{i}(t-1)+\sum_{j} P_{i j}^{k}(t) s(t) u_{j}(t)\right]_{k}$.

Lemma 7. Quantized communication static coordinate scheme DCDA algorithm: Consider the settings in Th. 1] scheme where the function is L-Lipschitz with respect to the $\ell_{2}$-norm. Furthermore define

$$
\nu(t)=\max _{k} \sum_{r=0}^{t} s^{2}(r) \sigma_{2}\left(P^{k}\right)^{2(t-r+1)}
$$

Under these assumptions, the quantized communication static sharing DCDA is such that

$$
\begin{aligned}
& f\left(\hat{x}_{i}(T)\right)-f\left(x^{*}\right) \leq(6)+R \sqrt{\widehat{s^{2}}(T) \frac{\log 1 / \delta}{T}}+\sum_{t=1}^{T} \alpha(t-1) \\
& \times\left(\frac{2 s(t) L+s^{2}(t)}{n T}+\frac{3 L}{T} \sqrt{2 \nu(t) \log (2 T n d / \delta)}\right) .
\end{aligned}
$$




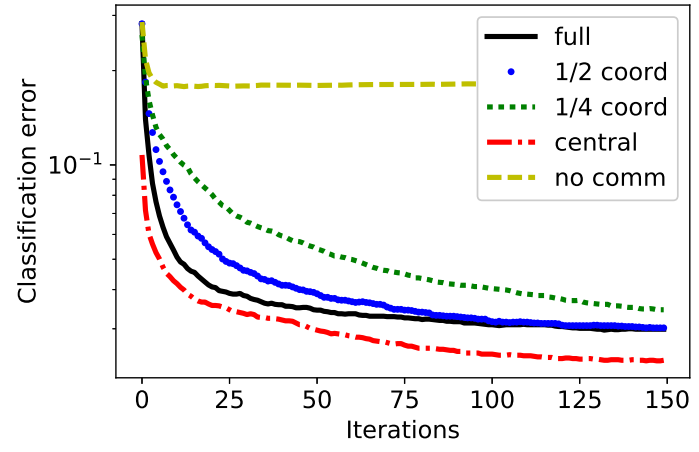

Fig. 1: Classification performance with distributed SVM

\section{NUMERICAL SIMULATIONS}

For numerical simulations, we consider the scenario in which the function $f_{i}(x)$ arises from evaluating a common loss function $\ell$ over a set of $m$ local measurements $\left\{z_{i j}\right\}_{j=1}^{m}$ : correspondingly we have

$$
F(x)=\sum_{i=1}^{n} f_{i}(x)=\sum_{i=1}^{n} \sum_{j=1}^{m} \ell\left(x, z_{i j}\right) .
$$

Support Vector Machine (SVM): In the first case, we look at using support vector machines for classification. Each datapoint at a local node consists of a label $l_{i j}$ uniformly drawn from $\{-1,1\}$ and the data point $z_{i j} \stackrel{\text { iid }}{\sim} \mathcal{N}\left(\mu_{l_{i j}}, \Sigma\right)$. The linear SVM algorithm finds a hyperplane that separates data drawn from the two distributions with the maximum margin

$$
\ell\left(x,\left(z_{i j}, l_{i j}\right)\right)=\frac{1}{2 d}\|x\|_{2}^{2}+C \sum_{j=1}^{m} \max \left(1-l_{i j} x^{T} z_{i j} ; 0\right) .
$$

In Fig. 1 we plot the simulation results for $X \in \mathbb{R}^{30}, n=10$ and $m=10$ and full network connectivity. At each instant in time, the nodes collectively sample a certain fraction $f$ of their coordinates to share. For this scenario, we compare the

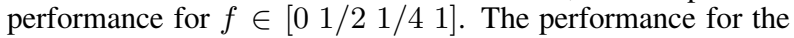
centralized SVM algorithm is also plotted for comparison. As it can be observed, without any communication, nodes reach a suboptimal solution. To reach $90 \%$ classification accuracy rate, nodes take twice as long if they share only half their coordinates. Finally, note the small loss in performance between the fully centralized scheme to the decentralized one.

Linear Regression: Next, we investigate the effect of noisy communication and stochastic gradient in the DCDA algorithm for the classic linear regression problem. We consider the case where $x \in \mathbb{R}^{30}, n=10$ and $m=20$ and a fully connected network. Note that local measurements consist of random normal measurement vectors $a_{i j}$ and the measurement $z_{i j}$. For the noisy communication scenario, each node observes $z_{i j}=$ $A_{i j} x+n_{i j}$, for $n_{i} \stackrel{\text { iid }}{\sim} \mathcal{N}(0, \Sigma)$ and $f_{i}(x)=\ell\left(x,\left(A_{i j}, z_{i j}\right)\right)=$ $\frac{1}{2}\left\|A_{i}: x-z_{i:}\right\|_{2}^{2}$ (with a slide abuse of notation). In the stochastic gradient case, nodes form mini-batches of size 4 as opposed to using all 20 data points for each iteration. As can be seen in Fig. 2, there is minimal loss in performance from using stochastic gradients or when additive noise is added to the nodes being shared. This suggests that cheaper computation using stochastic gradients, or quantization effects creating additive noise may not significantly alter performance.

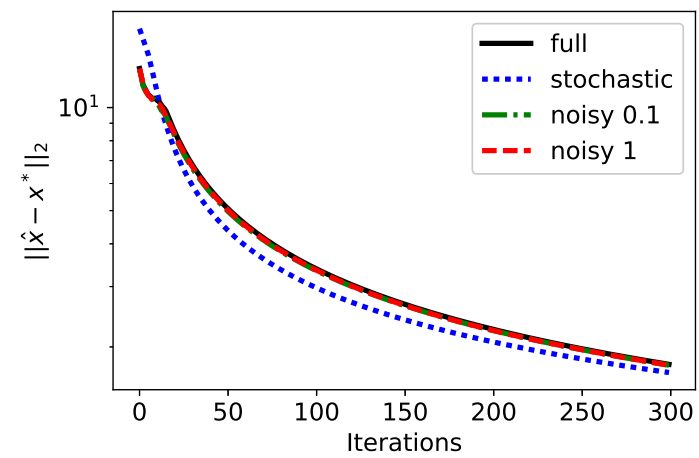

Fig. 2: Impact of stochastic gradients, noisy communications on performance of DCDA for linear regression

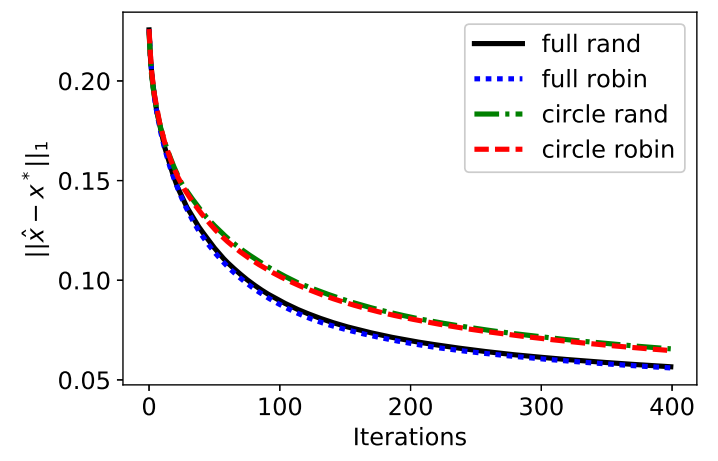

Fig. 3: Robust regression performance.

Robust Regression: Finally, we consider the robust regression problem in which each node observes $z_{i j}=A_{i j} x+$ $\left(1-b_{i j}\right) o_{i j}+b_{i j} n_{i j}$ where $x$ is in the unit simplex $\left([x]_{i} \geq\right.$ $\left.0,\|x\|_{1}=1\right), b_{i j}$ is a binomial noise that modulates between a large outlier Gaussian noise $o_{i j}$ or smaller additive Gaussian noise $n_{i j}$. The $\ell_{1}$ penalty is used as the loss function or $f_{i}(x)=\ell\left(x,\left(A_{i j}, z_{i j}\right)\right)=\left\|A_{i:} x-z_{i:}\right\|_{1}$. For this problem, we consider an entropic proximal function that ensures that the estimate is in the probability simplex and minimize the $\ell_{1}$ norm. In the simulation, we compare the round robin scheme and the randomized scheme where the amount of communication is kept equal. Both these schemes are compared for the fully connected network as well as a circle topology where nodes are connected to the closest neighbor on each side. Estimate $x \in \mathbb{R}^{20}$ and $m, n=10$. The performance is presented in Fig. 3 The performance of the fully connected layer is better than the circle topology because more communication is taking place, allowing estimates to quickly travel through the network. No significant difference in performance between the randomized and round robin schemes is observed.

\section{CONCLUSION}

We considered the problem of distributed optimization with limited communication where nodes collectively solve a convex optimization problem but have a limitation on the transmission among neighbors. We proposed a distributed coordinate dual averaging algorithm for this problem and analyzed its performance. We showed that the time required to achieve a fixed accuracy would double if the rate limitation in messages between nodes were halved. We also show convergence in the scenario of stochastic gradients, noisy and quantized communication. 


\section{A. REFERENCES}

[1] J. N. Tsitsiklis, "Problems in decentralized decision making and computation." Massachusetts Institute of Tech Cambridge Lab for Information and Decision Systems, Tech. Rep., 1984.

[2] A. Nedic and A. Ozdaglar, "Distributed subgradient methods for multi-agent optimization," IEEE Transactions on Automatic Control, vol. 54, no. 1, pp. 48-61, 2009.

[3] R. Olfati-Saber and R. M. Murray, "Consensus problems in networks of agents with switching topology and timedelays," IEEE Transactions on automatic control, vol. 49, no. 9, pp. 1520-1533, 2004.

[4] J. Wang and N. Elia, "A control perspective for centralized and distributed convex optimization," in Decision and Control and European Control Conference (CDCECC), 2011 50th IEEE Conference on. IEEE, 2011, pp. 3800-3805.

[5] I. Lobel and A. Ozdaglar, "Distributed subgradient methods for convex optimization over random networks," IEEE Transactions on Automatic Control, vol. 56, no. 6, p. 1291, 2011.

[6] P. Yi and Y. Hong, "Quantized subgradient algorithm and data-rate analysis for distributed optimization," IEEE Transactions on Control of Network Systems, vol. 1, no. 4, pp. 380-392, 2014.

[7] J. Liu, S. J. Wright, C. Ré, V. Bittorf, and S. Sridhar, "An asynchronous parallel stochastic coordinate descent algorithm," The Journal of Machine Learning Research, vol. 16, no. 1, pp. 285-322, 2015.

[8] Y. Nesterov, "Primal-dual subgradient methods for convex problems," Mathematical programming, vol. 120, no. 1, pp. 221-259, 2009.

[9] J. C. Duchi, A. Agarwal, and M. J. Wainwright, "Dual averaging for distributed optimization: Convergence analysis and network scaling," IEEE Transactions on Automatic control, vol. 57, no. 3, pp. 592-606, 2012.

[10] A. Agarwal and J. C. Duchi, "Distributed delayed stochastic optimization," in Advances in Neural Information Processing Systems, 2011, pp. 873-881.

[11] K. I. Tsianos and M. G. Rabbat, "Distributed dual averaging for convex optimization under communication delays," in American Control Conference (ACC), 2012. IEEE, 2012, pp. 1067-1072.

[12] K. Tsianos, S. Lawlor, and M. G. Rabbat, "Communication/computation tradeoffs in consensus-based distributed optimization," in Advances in neural information processing systems, 2012, pp. 1943-1951.

[13] E. Wei and A. Ozdaglar, "Distributed alternating direction method of multipliers," 2012.

[14] S. Boyd, N. Parikh, E. Chu, B. Peleato, J. Eckstein et al., "Distributed optimization and statistical learning via the alternating direction method of multipliers," Foundations and Trends ${ }^{\circledR}$ in Machine learning, vol. 3, no. 1, pp. 1122, 2011.

[15] W. Shi, Q. Ling, K. Yuan, G. Wu, and W. Yin, "On the linear convergence of the admm in decentralized consensus optimization." IEEE Trans. Signal Processing, vol. 62, no. 7, pp. 1750-1761, 2014.

[16] R. Zhang and J. Kwok, "Asynchronous distributed admm for consensus optimization," in International Conference on Machine Learning, 2014, pp. 1701-1709. 


\section{APPENDIX}

\section{Appendix A: Proof of Th. 1}

In this section prove the result in Th. 1 on the convergence of the DCDA algorithm. In our analysis of the DCDA algorithm, we employ techniques similar to those used in [9] to study the convergence of the DDA algorithm.

Before proceeding with the proof, let us introduce the auxiliary sequence $y(t)$ as the projection of $\bar{z}(t)$ using the operator $\Pi_{\psi, \alpha(t)}$, that is

$$
y(t)=\Pi_{\psi, \alpha(t-1)}(\bar{z}(t)) .
$$

An important consequence of double symmetric stochastic nature of the matrices $P^{k}$ is a particularly simple evolution of the variable $\bar{z}(t)$ as

$$
\begin{aligned}
{[\bar{z}(t+1)]_{k} } & =\frac{1}{n} \sum_{i=1}^{n} \sum_{j=1}^{n} P_{i j}^{k}\left[z_{j}(t)\right]_{k}+\left[g_{i}(t)\right]_{k} \\
& =\frac{1}{n} \sum_{j=1}^{n}\left[z_{j}(t)\right]_{k} \sum_{i=1}^{n} P_{i j}^{k}+\frac{1}{n} \sum_{i=1}^{n}\left[g_{i}(t)\right]_{k} \\
& =[\bar{z}(t)]_{k}+[\bar{g}(t)]_{k} .
\end{aligned}
$$

As for the result in [9 Th. 1], convergence of the local estimates to the global optimal is in terms of time averages $\hat{x}_{i}(t)$ and $\hat{y}(t)$ as Jensen's inequality can be used to show that

$$
f_{i}\left(\hat{x}_{i}(T)\right) \leq \frac{1}{T} \sum_{t^{\prime}=1}^{T} f_{i}\left(x_{i}(t)\right) .
$$

Paralleling the analysis in [9], we bound the error estimate as

$$
\begin{aligned}
& f\left(\hat{x}_{i}(T)\right)-f\left(x^{*}\right) \leq f(\hat{y}(T))-f\left(x^{*}\right)+L\left\|\hat{x}_{i}(T)-\hat{y}(T)\right\| \\
& \leq \frac{1}{T n} \sum_{t=1}^{T} \sum_{j=1}^{n} f_{j}\left(x_{j}(t)\right)-f_{j}\left(x^{*}\right)+\frac{1}{T n} \sum_{t=1}^{T} \sum_{j=1}^{n} f(y(t))-f_{j}\left(x_{j}(t)\right)+\frac{L}{T} \sum_{t=1}^{T}\left\|x_{i}(t)-y(t)\right\| \\
& \leq \frac{1}{T n} \sum_{t=1}^{T} \sum_{j=1}^{n} f_{j}\left(x_{j}(t)\right)-f_{j}\left(x^{*}\right)+\frac{L}{T n} \sum_{t=1}^{T} \sum_{j=1}^{n}\left\|y(t)-x_{j}(t)\right\|+\frac{L}{T} \sum_{t=1}^{T}\left\|x_{i}(t)-y(t)\right\| \\
& \leq \frac{1}{T n} \underbrace{\sum_{t=1}^{T} \sum_{j=1}^{n} f_{j}\left(x_{j}(t)\right)-f_{j}\left(x^{*}\right)}_{\Gamma} \\
&+\frac{L}{T n} \sum_{t=1}^{T} \sum_{j=1}^{n} \alpha(t-1)\left\|\bar{z}(t)-z_{j}(t)\right\|_{*}+\frac{L}{T} \sum_{t=1}^{T} \alpha(t-1)\left\|\bar{z}(t)-z_{i}(t)\right\|_{*},
\end{aligned}
$$

where the inequality in $19 \mathrm{a}$ follows by the Lipschitz property of $f$. Equations $19 \mathrm{c}$ and $19 \mathrm{~b}$ ) follow by the Lipschitz property of the projection operator as shown in the next lemma.

Lemma 1. [9. Lem. 5] $\Pi_{\psi, \alpha}(z)$ is $\alpha$-Lipschitz with respect to the dual norm. 
Next, we bound the term $\Gamma$ in $19 \mathrm{c}$ :

$$
\begin{aligned}
\Gamma & =\sum_{t=1}^{T} \sum_{j=1}^{n} f_{j}\left(x_{j}(t)\right)-f_{j}\left(x^{*}\right) \\
& \leq \sum_{t=1}^{T} \sum_{i=1}^{n}\left\langle g_{j}(t), x_{j}(t)-x^{*}\right\rangle \\
& =n \underbrace{\sum_{t=1}^{T}\left\langle\bar{g}(t), y(t)-x^{*}\right\rangle}_{\Psi_{1}} \\
& +\underbrace{\sum_{t=1}^{T} \sum_{i=1}^{n}\left\langle g_{j}(t), x_{j}(t)-y(t)\right\rangle}_{\Psi_{2}},
\end{aligned}
$$

where (20b) follows from the definition of subgradient. Let us first bound $\Psi_{2}$ in 20d). As the function $f_{j}$ is L-Lipschitz, the gradient is bounded in the dual norm, i.e. $\left\|g_{j}\right\|_{*} \leq L$, the term $\left\langle g_{j}(t), x_{j}(t)-y(t)\right\rangle$ can be bounded using Cauchy-Schwarz inequality:

$$
\begin{aligned}
\Psi_{2} & \leq \sum_{t=1}^{T} \sum_{j=1}^{n}\left\|g_{j}(t)\right\|_{*}\left\|x_{j}(t)-y(t)\right\| \\
& \leq L \sum_{t=1}^{T} \sum_{j=1}^{n}\left\|\Pi_{\psi, \alpha(t-1)}\left(z_{j}(t)\right)-\Pi_{\psi, \alpha(t-1)}(\bar{z}(t))\right\| \\
& \leq L \sum_{t=1}^{T} \sum_{j=1}^{n} \alpha(t-1)\left\|\bar{z}(t)-z_{j}(t)\right\|_{*}
\end{aligned}
$$

where $21 \mathrm{~b}$ follows from Lem. 1 Next, we bound the term $\Psi_{1}$ in $20 \mathrm{c}$ : this portion of the proof is similar to the bound in 9 . (20)-(21)]. Starting from the definition of $y(t)$, we write

$$
\begin{aligned}
y(t) & =\Pi_{\psi, \alpha(t-1)}(\bar{z}(t)) \\
& =\operatorname{argmin}_{x \in \mathcal{X}}\langle\bar{z}(t), x\rangle+\frac{1}{\alpha(t-1)} \psi(x) \\
& =\operatorname{argmax}_{x \in \mathcal{X}}\langle-\bar{z}(t), x\rangle-\frac{1}{\alpha(t-1)} \psi(x) .
\end{aligned}
$$

Let us next use the dual function $\psi_{\alpha}^{*}(z)=\sup _{x}\langle z, x\rangle-\frac{1}{\alpha} \psi(x)$ to find an upper bound $\Psi_{1}$. Since the set $\mathcal{X}$ is closed and convex and $\psi$ is convex, we have that the supremum is attained. In other words, we have,

$$
\psi_{\alpha(t-1)}^{*}(-\bar{z}(t))=\langle-\bar{z}(t), y(t)\rangle-\frac{1}{\alpha(t-1)} \psi(y(t)) .
$$

Accordingly, for arbitrary $u \in \mathcal{X}$, we have

$$
\begin{aligned}
\psi_{\alpha(t-1)}^{*}(u) & \geq\langle u, y(t)\rangle-\frac{1}{\alpha(t-1)} \psi(y(t)) \\
& \geq\langle-\bar{z}(t), y(t)\rangle-\frac{1}{\alpha(t-1)} \psi(y(t))+\langle y(t), u+\bar{z}(t)\rangle \\
& =\psi_{\alpha(t-1)}^{*}(-\bar{z}(t))+\langle y(t), u+\bar{z}(t)\rangle,
\end{aligned}
$$

which shows that $\nabla \psi_{\alpha(t-1)}^{*}(-\bar{z}(t))=y(t)$. Next, from the strong convexity and positivity of $\psi$ we have that, for any $t \in[0,1]$, there exists $c \in[0, t]$ such that

$$
\psi_{\alpha}^{*}(z-t g)=\psi_{\alpha}^{*}(z)-t\left\langle\nabla \psi_{\alpha}^{*}(z-c g), g\right\rangle .
$$


Using $t=1$ and rearranging,

$$
\begin{aligned}
\psi_{\alpha}^{*}(z-g) & =\psi_{\alpha}^{*}(z)-\left\langle\nabla \psi_{\alpha}^{*}(z), g\right\rangle-\left\langle\nabla \psi_{\alpha}^{*}(z)-\nabla \psi_{\alpha}^{*}(z-c g), g\right\rangle \\
& \leq \psi_{\alpha}^{*}(z)-\left\langle\nabla \psi_{\alpha}^{*}(z), g\right\rangle+\|g\|_{*}\left\|\nabla \psi_{\alpha}^{*}(z)-\nabla \psi_{\alpha}^{*}(z-c g)\right\| \\
& \leq \psi_{\alpha}^{*}(z)-\left\langle\nabla \psi_{\alpha}^{*}(z), g\right\rangle+\alpha\left\|_{g}\right\|_{*}^{2} .
\end{aligned}
$$

Using (23) for $z=\bar{z}(t)$ and $g=\bar{g}(t)$ yields

$$
\begin{aligned}
\psi_{\alpha(t)}^{*}(-\bar{z}(t)-\bar{g}(t)) & \leq \psi_{\alpha(t-1)}^{*}(-\bar{z}(t)-\bar{g}(t)) \\
& \leq \psi_{\alpha(t-1)}^{*}(-\bar{z}(t))-\langle y(t), \bar{g}(t)\rangle+\alpha(t-1)\|\bar{g}(t)\|_{*}^{2},
\end{aligned}
$$

and thus

$$
\langle y(t), \bar{g}(t)\rangle \leq \psi_{\alpha(t-1)}^{*}(-\bar{z}(t))-\psi_{\alpha_{t}}^{*}(-\bar{z}(t+1))+\alpha(t-1)\|\bar{g}(t)\|_{*}^{2} .
$$

From the definition of the dual function, we have

$$
\begin{aligned}
\left\langle-\bar{z}(T+1), x^{*}\right\rangle-\frac{1}{\alpha(t)} \psi\left(x^{*}\right) & \leq \psi_{\alpha(t)}^{*}(\bar{z}(T+1)) \\
\Rightarrow\left\langle\bar{z}(T+1),-x^{*}\right\rangle & \leq \frac{1}{\alpha(t)} \psi\left(x^{*}\right)+\psi_{\alpha(t)}^{*}(\bar{z}(T+1)) .
\end{aligned}
$$

Combining 25 and 24, we obtain a bound on $\Psi$ in $20 \mathrm{c}$ as

$$
\begin{aligned}
\Psi_{1}=\sum_{t=1}^{T}\left\langle\bar{g}(t), y(t)-x^{*}\right\rangle & =\sum_{t=1}^{T}\left(\langle\bar{g}(t), y(t)\rangle+\left\langle\bar{g}(t),-x^{*}\right\rangle\right) \\
& \leq \sum_{t=1}^{T} \alpha(t-1)\|\bar{g}(t)\|_{*}^{2}+\frac{1}{\alpha(t)} \psi\left(x^{*}\right) .
\end{aligned}
$$

Substituting the bound in 26 and $21 \mathrm{~b}$ ) in $19 \mathrm{c}$ yields the bound in (5).

\section{Appendix B: Proof of Lem. 2}

In the static sharing scheme, each coordinate of the optimization variable follows a different but constant graph, $P^{k}(t)=P^{k}$, for all $t \in \mathbb{N}$. In the analysis of this scheme, we rely on the result of Th. [1] and bound the term $\left[\bar{z}(t)-z_{i}(t)\right]_{k}$ using the properties of the communication protocol. To this end, we define

$$
\begin{aligned}
\Phi^{k}(t, s) & =P^{k}(t) P^{k}(t-1) \ldots P^{k}(s) \\
& =\prod_{j=t}^{s} P^{k}(j),
\end{aligned}
$$

so that the evolution of the dual variable can be expressed recursively as

$$
\left[z_{i}(t+1)\right]_{k}=\sum_{j=1}^{n}\left[\Phi^{k}(t, s)\right]_{i j}\left[z_{j}(s)\right]_{k}+\left[g_{i}(t)\right]_{k}+\sum_{r=s+1}^{t} \sum_{j=1}^{n}\left[\Phi^{k}(t, r)\right]_{i j}\left[g_{j}(r-1)\right]_{k},
$$

for any $0 \leq s<t$. We set the starting value $s=0, z_{i}(s)=0$. Now we can see

$$
\left[\bar{z}(t)-z_{i}(t)\right]_{k}=\sum_{r=1}^{t-1} \sum_{j=1}^{n}\left(1 / n-\left[\Phi^{k}(t-1, r)\right]_{i j}\right)\left[g_{j}(r-1)\right]_{k}+\frac{1}{n} \sum_{j=1}^{n}\left[g_{j}(t-1)-g_{i}(t-1)\right]_{k} .
$$

In the following, we also resort to the following inequality from Perron-Frobenius theory for double-stochastic matrices: for any $x$ within the $n$-dimensional probability simplex:

$$
\left\|\left(P^{k}\right)^{t} x-\frac{\mathbf{1}}{n}\right\|_{1} \leq \sqrt{n}\left\|\left(P^{k}\right)^{t} x-\frac{\mathbf{1}}{n}\right\|_{2} \leq \sqrt{n} \sigma_{2}\left(P^{k}\right)^{t},
$$


where $\sigma_{2}(\cdot)$ is the second singular value of a matrix. We are now ready to bound the term $\left\|\bar{z}(t)-z_{i}(t)\right\|_{*}$ :

$$
\begin{aligned}
\left\|\bar{z}(t)-z_{i}(t)\right\|_{*} & \leq \sum_{r=1}^{t-1} \sum_{j=1}^{n}\left\|g_{j}(r-1)\right\|_{*} \max _{k}\left|\frac{1}{n}-\left[\Phi^{k}(t-1, r)\right]_{i j}\right|+\frac{1}{n} \sum_{j=1}^{n}\left\|g_{j}(t-1)-g_{i}(t-1)\right\|_{*} \\
& \leq \sum_{r=1}^{t-1} L \sum_{k}\left\|\frac{\mathbf{1}}{n}-\Phi^{k}(t-1, r)_{i}\right\|_{1}+2 L
\end{aligned}
$$

When $t-r \geq \Delta_{t}=\frac{\log \sqrt{n} d T / \epsilon}{-\log \min _{k} \sigma_{2}\left(P^{k}\right)}$, we have $\sum_{k}\left\|\frac{1}{n}-\Phi^{k}(t-1, r)_{i}\right\|_{1} \leq \frac{1}{T}$. Else, this sum would be less than $2 \mathrm{~min}(d, n)$. We see this as,

$$
\begin{aligned}
\sum_{j=1}^{n} \max _{k}\left|\frac{1}{n}-\left[\Phi^{k}(t-1, r)\right]_{i j}\right| & \leq \sum_{k}\left\|\frac{\mathbf{1}}{n}-\Phi^{k}(t-1, r)_{i}\right\|_{1} \\
& =\sum_{k}\left\|\frac{\mathbf{1}}{n}\right\|_{1}+\left\|\Phi^{k}(t-1, r)_{i}\right\|_{1}=2 d
\end{aligned}
$$

and

$$
\sum_{j=1}^{n} \max _{k}\left|\frac{1}{n}-\left[\Phi^{k}(t-1, r)\right]_{i j}\right| \leq \sum_{j=1}^{n} \frac{1}{n}+\max _{k}\left[\Phi^{k}(t-1, r)\right]_{i j}=2 n .
$$

Thus we can bound the sum

$$
\begin{aligned}
\left\|\bar{z}(t)-z_{i}(t)\right\|_{*} & \leq \sum_{r=t-\Delta_{t}}^{t-1} L \sum_{k}\left\|\frac{\mathbf{1}}{n}-\Phi^{k}(t-1, r)_{i}\right\|_{1}+\sum_{r=1}^{t-\Delta_{t}} \sum_{k}\left\|\frac{\mathbf{1}}{n}-\Phi^{k}(t-1, r)_{i}\right\|_{1}+2 L \\
& \leq 2 \min (d, n) L \frac{\log \sqrt{n} d T / \epsilon}{-\log \min _{k} \sigma_{2}\left(P^{k}\right)}+L+2 L \\
& =L\left(2 \min (d, n) \frac{\log \sqrt{n} d T / \epsilon}{-\log \min _{k} \sigma_{2}\left(P^{k}\right)}+3\right) .
\end{aligned}
$$

Remark. Note the factor of $\min (d, n)$ in $31 \mathrm{c}$ : this can be reduced if multiple coordinates follow the same weight function. In an extreme case, this factor of $d$ would be removed if all coordinates are transmitted at each point in time.

\section{Appendix C: Proof of Lem. 3}

In the analysis round robin scheme, $m$ of of the $d$ components of the dual vector are shared at each point in time. Accordingly, any given coordinate is shared among a set of nodes every $\kappa=d / m$ time instances. For simplicity, assume that $m$ divides $d$ : if this is not the case, one can upper bound performance considering $m^{\prime}=d\lfloor m d\rfloor$.

At the time instant $T=l \kappa+\tau$, the coordinates $\left[k_{m+1} \ldots k_{\tau(m+1)}\right]$ are transmitted by each node over the graph $P$. Using the notation in 28 and given that $m$ divides $d$, we write

$$
\begin{aligned}
\Phi^{k_{h}}(T, r) & =P^{k_{h}}(T) \cdots P^{k_{h}}(r) \\
& = \begin{cases}P^{\left\lfloor\frac{T-r}{\kappa}\right\rfloor} & r<T-\left(\left\lfloor\frac{T-r}{\kappa}\right\rfloor+1\right) \kappa+\tau \\
P^{\left\lfloor\frac{T-r}{\kappa}\right\rfloor+1} & \text { otherwise. }\end{cases}
\end{aligned}
$$

We can now see that

$$
\begin{aligned}
\left\|\bar{z}(T+1)-z_{i}(T+1)\right\|_{*} & \leq \sum_{r=1}^{T} \sum_{j=1}^{n}\left\|g_{j}(r-1)\right\|_{*} \max _{h}\left|1 / n-\left[\Phi^{k_{h}}(T, r)\right]_{i j}\right|+\frac{1}{n} \sum_{j=1}^{n}\left\|g_{j}(T)-g_{i}(T)\right\|_{*} \\
& \leq \sum_{r=1}^{T} \sum_{j=1}^{n}\left\|g_{j}(r-1)\right\|_{*}\left(\left|1 / n-\left[P^{\left\lfloor\frac{T-r}{\kappa}\right\rfloor}\right]_{i j}\right|+\left|1 / n-\left[P^{\left\lfloor\frac{T-r}{\kappa}\right\rfloor+1}\right]_{i j}\right|\right)+\frac{1}{n} \sum_{j=1}^{n}\left\|g_{j}(T)-g_{i}(T)\right\|_{*} \\
& \leq L \sum_{r=1}^{T}\left\|1 / n-\left[P^{\left\lfloor\frac{T-r}{\kappa}\right\rfloor}\right]_{i}\right\|_{1}+\left\|1 / n-\left[P^{\left\lfloor\frac{T-r}{\kappa}\right\rfloor+1}\right]_{i}\right\|_{1}+2 L
\end{aligned}
$$


When $r<T-\Delta_{t} \kappa$ where $\Delta_{t}=\frac{\log 2 \sqrt{n} T}{-\log \sigma_{2}(P)}$, we have

$$
\left\|1 / n-\left[P^{\left\lfloor\frac{T-r}{\kappa}\right\rfloor}\right]_{i}\right\|_{1}+\left\|1 / n-\left[P^{\left\lfloor\frac{T-r}{\kappa}\right\rfloor+1}\right]_{i}\right\|_{1} \leq \frac{1}{T},
$$

else,

$$
\left\|1 / n-\left[P^{\left\lfloor\frac{T-r}{\kappa}\right\rfloor}\right]_{i}\right\|_{1}+\left\|1 / n-\left[P^{\left\lfloor\frac{T-r}{\kappa}\right\rfloor+1}\right]_{i}\right\|_{1} \leq 4 .
$$

Thus the summation in $32 \mathrm{~b}$ ) is further loosened as

$$
\left\|\bar{z}(T+1)-z_{i}(T+1)\right\|_{*} \leq 4 L \frac{d \log 2 \sqrt{n} T}{-m \log \sigma_{2}(P)}+3 L .
$$

Recall also that $\log x \leq x-1$ for $x \in(0,1)$. Hence $1 /-\log \sigma_{2}(P) \leq 1 / 1-\sigma_{2}(P)$. Combining this observation with (33) yields the bound in (8).

\section{Appendix D: Proof of Lem. 4}

In the randomized communication scheme, nodes select a different subset of coordinates to transmit at each time step. This selection is assumed to be random and independent. This communication strategy can be implemented, for instance, by having nodes share a random seed and hence share the same random subset of coordinates. Another alternative is the one in which nodes do not share a random seed and decide at each time step independent of each other what coordinates to transmit.

We first prove the convergence of the random time-varying $\Phi(t, s)$ to $\mathbf{1 1}^{\top} / n$ : consider a $u(t)$ in the probability simplex evolving as $u(t+1)=P^{k}(t) u(t)$, then

$$
\begin{aligned}
\mathbb{E}[\langle u(t+1)-\mathbf{1} / n, u(t+1)-\mathbf{1} / n\rangle \mid u(t)] & =(u(t)-\mathbf{1} / n)^{\top} \mathbb{E}\left[P^{k}(t)^{2}\right](u(t)-\mathbf{1} / n) \\
& \leq\|u(t)-\mathbf{1} / n\|_{2}^{2} \lambda_{2}\left(\mathbb{E}\left[P^{k}(t)^{2}\right]\right),
\end{aligned}
$$

where, (35) follows because the leading eigenvalue and eigenvector of $\mathbb{E}\left[P^{k}(t)^{2}\right]$ are 1 and $\mathbf{1} / n$ respectively. Next, Chebyshev inequality can be used to derive the bound

$$
\begin{aligned}
\mathbb{P}\left(\|u(t)-\mathbf{1} / n\|_{2} \geq \epsilon \mid u(0)\right) & \leq \frac{\mathbb{E}\left[\left|u(t)-\mathbf{1} / n \|_{2}\right| u(0)\right]}{\epsilon^{2}} \\
& \leq \epsilon^{-2}\|u(0)-\mathbf{1} / n\|_{2}^{2} \mathbb{E}\left[P^{k}(t)^{2}\right]^{t}
\end{aligned}
$$

and thus we have

$$
\mathbb{P}\left(\left\|\Phi^{k}(t, s) e_{i}-\mathbf{1} / n\right\|_{2} \geq \epsilon\right) \leq \epsilon^{-2} \lambda_{2}\left(\mathbb{E}\left[P^{k}(t)^{2}\right]\right)^{t-s+1}
$$

Now, with $t-s>\Delta_{k}=\frac{\log T^{3} d^{3} n / \delta}{1-\lambda_{2}\left(\mathbb{E}\left[P^{k}(t)^{2}\right]\right)}$, we have that, with probability $1-\frac{\delta}{T d}$

$$
\begin{aligned}
\left\|\frac{1}{n}-\Phi^{k}(t-1, s) e_{i}\right\|_{1} & \leq \sqrt{n}\left\|\frac{1}{n}-\Phi^{k}(t-1, s) e_{i}\right\|_{2} \\
& \leq \sqrt{n}\left\|\frac{1}{n}-\Phi^{k}\left(t-1, t-\Delta_{k}\right) e_{i}\right\|_{2} \\
& \leq \frac{1}{T d},
\end{aligned}
$$

where the inequality in (36b) follow because, for any $s^{\prime}<s, \Phi\left(t-1, s^{\prime}\right)=\Phi\left(s, s^{\prime}\right) \Phi(t-1, s),\left\|\Phi\left(s, s^{\prime}\right)\right\|_{2} \leq 1$, and $\left\|\Phi\left(t-1, s^{\prime}\right) e_{i}-\mathbf{1} / n\right\|_{2} \leq\left\|\Phi\left(s, s^{\prime}\right)\right\|_{2}\left\|\Phi(t-1, s) e_{i}-\mathbf{1} / n\right\|_{2}$. Note that for $k \neq k^{\prime}, \mathbb{E}\left[P^{k}(t)^{2}\right]=\mathbb{E}\left[P^{k^{\prime}}(t)^{2}\right]$, so that the bound in (36) holds for any $k$ and and the same $\Delta_{k}=\Delta_{k^{\prime}}=\Delta$.

\section{Appendix E: Proof of Lem. 5}

In a stochastic gradient DCDA algorithm, each node does not have access to the gradient of its local function. For instance, if a node may use a fraction of its total data to compute a gradient. This provides a cheap but noisy estimate of the gradient. For this scenarios, we are again concerned with the convergence analysis as in Th. 1 For this reason, we consider the same bounding as in (19) and consider the additional terms arising from the presence of a stochastic gradient. 
The difference in analysis with respect to the derivation in App. A starts from $\Gamma$ in Eq. (20a):

$$
\begin{aligned}
\Gamma & =\sum_{t=1}^{T} \sum_{i=1}^{n} f_{i}\left(x_{i}(t)\right)-f_{i}\left(x^{*}\right) \\
& \leq \sum_{t=1}^{T} \sum_{i=1}^{n}\left\langle g_{i}^{\prime}(t), x_{i}(t)-x^{*}\right\rangle \\
& \leq \sum_{t=1}^{T} \sum_{i=1}^{n}\left\langle g_{i}(t), x_{i}(t)-x^{*}\right\rangle+\sum_{t=1}^{T} \sum_{i=1}^{n}\left\langle g_{i}(t)-g_{i}^{\prime}(t), x_{i}(t)-x^{*}\right\rangle
\end{aligned}
$$

The analysis of the first term proceed as in the analysis of of $20 \mathrm{~b}$ in App. A. The second term forms a martingale sequence as

$$
\mathbb{E}\left[\left\langle g_{i}(t)-g_{i}^{\prime}(t), x_{i}(t)-x^{*}\right\rangle \mid \mathcal{F}_{t-1}\right]=\left\langle g_{i}(t)-\mathbb{E}\left[g_{i}^{\prime}(t) \mid \mathcal{F}_{t-1}\right], x_{i}(t)-x^{*}\right\rangle=0
$$

and also bounded as

$$
\left\langle g_{i}(t)-g_{i}^{\prime}(t), x_{i}(t)-x^{*}\right\rangle \leq 2 L R
$$

give the assumption on the boundedness of $\left\|x-x^{\prime}\right\|$ in Ass. 1 As $\left\langle g_{i}(t)-g_{i}^{\prime}(t), x_{i}(t)-x^{*}\right\rangle$ is a bounded martingale sequence, we can apply the Azuma-Hoeffding inequality to obtain

$$
\mathbb{P}\left[\sum_{t=1}^{T} \sum_{i=1}^{n}\left\langle g_{i}(t)-g_{i}^{\prime}(t), x_{i}(t)-x^{*}\right\rangle \geq T n \epsilon\right] \leq \exp \left(-\frac{T \epsilon^{2}}{8 L^{2} R^{2}}\right) .
$$

Thus, with probability $1-\delta$, we have

$$
\frac{1}{n T} \sum_{t=1}^{T} \sum_{i=1}^{n}\left\langle g_{i}(t)-g_{i}^{\prime}(t), x_{i}(t)-x^{*}\right\rangle \leq L R \sqrt{\frac{-8 \log \delta}{T}} .
$$

The remaining terms in (19) are bounded as in App. A, so that the inequality in (9) is obtained as (5) plus the additional term in 39.

\section{Appendix F: Proof of Lem. 6}

In this appendix, we prove convergence of the noisy communication DCDA algorithm for the static staring communication strategy. The proof is an adaptation of the proof of Lem. 2 that accounts for the additional additive noise term. Similarly to the derivation in App. E, the result in Th. 1 can be leveraged to study the scenario of noisy communications in two steps: (i) bound the additional terms arising in $\Gamma$ because of the presence of the additive noise as in [37), and (ii) bound the convergence of the term $\left\|\bar{z}(t)-z_{i}(t)\right\|_{*}$ under the assumption it the statement of Lem. 6 on the choice of norm and communication startegy.

Let us begin from the bounding of $\left\|\bar{z}(t)-z_{i}(t)\right\|_{*}$ : note that the update of the dual variable $z_{i}$ is now obtained as

$$
\begin{aligned}
{\left[z_{i}(t+1)\right]_{k} } & =\sum_{j=1}^{n} P_{i j}^{k}(t)\left[z_{j}(t)+n_{i j}(t)\right]_{k}+\left[g_{i}(t)\right]_{k} \\
& =\sum_{j=1}^{n} P_{i j}^{k}(t)\left[z_{j}^{p}(t)+n_{j}(t)+n_{i j}(t)\right]_{k}+\left[g_{i}(t)\right]_{k} \\
& \left.=\sum_{j=1}^{n} P_{i j}^{k}(t)\left[z_{j}(t)^{p}\right]_{k}+\left[g_{i}(t)\right]_{k}+\sum_{j=1}^{n} P_{i j}^{k}\left[n_{j}(t)+n_{i j}(t)\right]_{k}\right] \\
& =\left[z_{i}^{p}(t+1)\right]_{k}+\left[n_{i}(t+1)\right]_{k},
\end{aligned}
$$

where, in 40b we define $z_{j}^{p}(t)$ as the dual variable evolution in the noiseless case as in (17) (save for the spacial average), while $n_{i}(t)$ is defined the total accumulated noise on the dual variable $z_{i}(t)$, through the recursion

$$
\left[n_{i}(t+1)\right]_{k}=\sum_{j=1}^{n} P_{i j}^{k}\left[n_{j}(t)_{k}+n_{i j}(t)\right]
$$


Note that the recursion in (41) can be reformulated using the notation in 27) as

$$
\left[n_{i}(t+1)\right]_{k}=\sum_{r=1}^{t+1} \sum_{j=1}^{n} \Phi_{i j}^{k}(t+1, r)\left[n_{i j}(r-1)\right]_{k} .
$$

Under the assumption that the noise $n_{i j}(t)$ has independent zero-mean sub-Gaussian components (can be weakened to rotationally symmetric vector with sub-Gaussian tails) of power $\gamma^{2} / d$. Additionally,

$$
\begin{aligned}
\mathbb{E}\left[z_{i}(t)-z_{i}^{p}(t)\right] & =0 \\
\mathbb{E}\left[\bar{z}(t)-z_{i}(t)-\bar{z}^{p}(t)+z_{i}^{p}(t)\right] & =0 .
\end{aligned}
$$

Next, let us define $\Delta z_{i}(t)=\bar{z}(t)-z_{i}(t)$ and $\Delta z_{i}^{p}(t)=\bar{z}^{p}(t)-z_{i}^{p}(t)$ so that their difference, $\Delta z_{i}(t+1)-\Delta z_{i}^{p}(t+1)$ is also sub-Gaussian and can be expressed as

$$
\left[\Delta z_{i}(t+1)-\Delta z_{i}^{p}(t+1)\right]_{k}=\sum_{r=1}^{t+1} \sum_{j=1}^{n}\left(\frac{1}{n}-\Phi_{i j}^{k}(t+1, r)\right)\left[n_{i j}(r-1)\right]_{k}
$$

so that the variance proxy $\sigma^{2}(\cdot)$ is obtained as

$$
\begin{aligned}
\sigma^{2}\left(\left[\Delta z_{i}(t+1)-\Delta z_{i}^{p}(t+1)\right]_{k}\right) & \leq \frac{\gamma^{2}}{d} \sum_{r=1}^{t+1}\left\|\Phi_{i}^{k}(t+1, r)-\frac{\mathbf{1}}{n}\right\|_{2}^{2} \\
& \leq \frac{\gamma^{2}}{d\left(1-\sigma_{2}\left(P^{k}\right)^{2}\right)}
\end{aligned}
$$

where, in (47), we have used the fact that $\left\|\Phi_{i j}^{k}(t, r)-\mathbf{1} / n\right\|_{2}=\left\|\left(P^{k}\right)^{t-r+1} e_{i}-\mathbf{1} / n\right\|_{2} \leq \sigma_{2}\left(P^{k}\right)^{t-r+1}$. Using the deviation bound for sub-Gaussian variables

$$
\mathbb{P}(|X-\mathbb{E}[X]| \geq \epsilon) \leq 2 \exp \left(-\epsilon^{2} / 2 \sigma^{2}\right)
$$

for $X$ sub-Gaussian with with variance proxy parameter $\sigma^{2}$, we conclude that, with probability $1-\delta$

$$
\left|\left[\Delta z_{i}(t)-\Delta z_{i}^{p}(t)\right]_{k}\right| \leq \gamma \sqrt{\frac{2 \log 2 T d n / \delta}{d\left(1-\sigma_{2}\left(P^{k}\right)^{2}\right)}} \quad \forall t \leq T, i \in[n], k \in[d] .
$$

Thus with probability $1-\delta$, we have

$$
\frac{1}{T} \sum_{t=1}^{T}\left\|\bar{z}(t)-z_{i}(t)\right\|_{*} \leq \frac{1}{T} \sum_{t=1}^{T}\left\|\bar{z}^{p}(t)-z_{i}^{p}(t)\right\|_{*}+\frac{1}{T} \sqrt{\frac{2 \gamma^{2} \log 2 T n d / \delta}{d\left(1-\max _{k} \sigma_{2}\left(P^{k}\right)^{2}\right)}}\|\mathbf{1}\|_{*},
$$

Let us next return to the bounding of $\Gamma$ as in 20c): in the scenario of noisy communication this term can be bounded similarly to the stochastic gradient scenario by letting $g_{i}^{\prime}(t)=g_{i}(t)+\sum_{j} P_{i j}^{k}(t) n_{i j}(t)$. With this definition, the term $\Psi_{1}$ in $(20 \mathrm{c})$ can be bounded as

$$
\begin{aligned}
& \sum_{t=1}^{T}\left\langle\overline{g^{\prime}}(t), y(t)-x^{*}\right\rangle \\
& =\sum_{t=1}^{T}\left\langle\bar{g}(t)+\bar{n}(t), y(t)-x^{*}\right\rangle-\sum_{t=1}^{T}\left\langle\bar{n}(t), y(t)-x^{*}\right\rangle \\
& \leq \sum_{t=1}^{T} \alpha(t-1)\left(\|\bar{g}(t)+\bar{n}(t)\|_{*}^{2}\right)+\frac{1}{\alpha(T)} \psi\left(x^{*}\right)-\sum_{t=1}^{T}\left\langle\bar{n}(t), y(t)-x^{*}\right\rangle . \\
& \leq \sum_{t=1}^{T} \alpha(t-1)\left(\|\bar{g}(t)\|_{*}^{2}+\|\bar{n}(t)\|_{*}^{2}\right)+\frac{1}{\alpha(T)} \psi\left(x^{*}\right)+\sum_{t=1}^{T}\left\langle\bar{n}(t), x^{*}-y(t)+2 \alpha(t-1) \bar{g}(t)\right\rangle .
\end{aligned}
$$


Here, $\bar{n}(t)=\frac{1}{n} \sum_{i} \sum_{j} P_{i j}^{k}(t) n_{i j}(t)$. To proceed in the bounding of the term $\sum_{t=1}^{T} \alpha(t-1)\|\bar{n}(t)\|_{*}^{2}$, we utilize the assumption that $\|\cdot\|$ is the $\ell_{2}$-norm and that $\sup _{x, x^{\prime}}\left\|x-x^{\prime}\right\| \leq R$. Since $[\bar{n}(t)]_{k} \sim \mathcal{N}\left(0, \frac{\gamma^{2}}{d n}\right)$, we have that $\|\bar{n}(t)\|_{2}^{2}$ is a sub-exponential variable with parameters $\left(\frac{2 \gamma^{2}}{d \sqrt{n}}, \frac{4 \gamma^{2}}{n d}\right)$. Accordingly, $\sum_{t=1}^{T} \alpha(t-1)\|\bar{n}(t)\|_{2}^{2}$ is sub-exponential with parameters

$$
\left(\frac{2 \gamma^{2}}{d \sqrt{ } n} \sqrt{\sum_{t=1}^{T} \alpha^{2}(t-1)}, \max _{t>1} \frac{4 \gamma^{2} \alpha(t)}{n d}\right) .
$$

We now employ the concentration inequality for sub-exponential random variable $Z$ with parameters $(\nu, b)$ :

$$
\mathbb{P}(Z \geq \mathbb{E}[Z]+\epsilon) \leq \exp \left(-\frac{\epsilon^{2}}{2 \nu^{2}}\right) \quad \forall \epsilon \leq \frac{\nu^{2}}{b}
$$

We get that with probability greater than $1-\delta$ and large enough $T$ is

$$
\begin{aligned}
\frac{1}{T} \sum_{t=1}^{T} \alpha(t-1)\|\bar{n}(t)\|_{2}^{2} & \leq \frac{\gamma^{2}}{n d T}\left\{\sqrt{8 \sum_{t=1}^{T} \alpha^{2}(t-1)} \log 1 / \delta+\sum_{t=1}^{T} \alpha(t-1)\right\} \\
& \leq \frac{\gamma^{2}}{n d T}\left(\sqrt{8} \log \frac{1}{\delta}+1\right) \sum_{t=1}^{T} \alpha(t-1) .
\end{aligned}
$$

We can see that $\left\langle\bar{n}(t), x^{*}-y(t)+2 \alpha(t-1) \bar{g}(t)\right\rangle$ is sub-Gaussian with zero mean and $\mathrm{n}$ variance proxy parameter

$$
\sigma^{2}\left(\left\langle\bar{n}(t), x^{*}-y(t)+2 \alpha(t-1) \bar{g}(t)\right\rangle\right) \leq \frac{\gamma^{2}(R+2 L)^{2}}{n}
$$

so that, with probability $1-\delta$

$$
\left|\frac{1}{T} \sum_{t=1}^{T}\left\langle\bar{n}(t), x^{*}-y(t)+\alpha(t-1) \bar{g}(t)\right\rangle\right| \leq \gamma(R+2 L) \sqrt{\frac{2 \log \frac{1}{\delta}}{n T}} .
$$

Combining the penalties in (48), 50) and (51) we obtain the bound in (10).

\section{Appendix G: Proof of Lem. 7}

We finally come to the analysis of the performance of the DCDA algorithm with quantized communication in the static sharing scheme. Similarly to the derivation in 40c in App. F for the noisy communication scenario, the dual variable update can be rewritten as

$$
\begin{aligned}
{\left[z_{i}(t+1)\right]_{k} } & =\left[z_{i}(t)\right]_{k}+\sum_{j \in N^{k}(i)} P_{i j}^{k}\left[\left(z_{j}(t)-z_{j}(t-1)\right)+s(t) \Delta_{j}(k)\right]_{k}+\left[g_{i}(t)\right]_{k}-\left[g_{i}(t-1)\right]_{k} \\
& =\sum_{j \in N^{k}(i)} P_{i j}^{k}\left[z_{j}(t-1)\right]_{k}+\sum_{j \in N^{k}(i)} P_{i j}^{k}\left[\left(z_{j}(t)-z_{j}(t-1)\right)+s(t) \Delta_{j}(k)\right]_{k}+\left[g_{i}(t)\right]_{k} \\
& =\sum_{j \in N^{k}(i)} P_{i j}^{k}\left[z_{j}(t)\right]_{k}+\left[g_{i}(t)\right]_{k}+s(t) \sum_{j \in N^{k}(i)} P_{i j}^{k} \Delta_{j}(k) \\
& \left.=z_{i}^{p}(t+1)\right]_{k}+n_{i}(t+1),
\end{aligned}
$$

where, in 52a we have used the definition of the transmitted message and the dual variable update in (11) and (12) respective, and where we have defined $\Delta_{j}(t)$ as the quantization error, i.e.

$$
\left.\left[\Delta_{j}(t)\right]_{k}=u_{i}(t)\right]_{k}-\left\lfloor\frac{\left[z_{j}(t)\right]_{k}-\left[z_{j}(t-1)\right]_{k}}{s(t)}\right\rfloor,
$$

while, in $52 \mathrm{~b}$, we have defined $z_{i}^{p}(t)$ as in App. F as the value of the dual variable in the noiseless case. Also, similarly to App. $\mathrm{F}, n_{i}(t)$ as the total accumulated error between $z_{i}^{p}(t)$ and $z_{i}(t)$, obtained as

$$
\left[n_{i}(t+1)\right]_{k}=\sum_{j=1}^{n} P_{i j}^{k}\left[n_{i}(t)_{k}+\Delta_{j}(t)\right]
$$


Similarly to (42), $n_{i}(t+1)$ can be recursively expressed as

$$
\left[n_{i}(t+1)\right]_{k}=\sum_{r=1}^{t+1} \sum_{j=1}^{n} \Phi_{i j}^{k}(t+1, r)\left[\Delta_{j}(r-1)\right]_{k} .
$$

The expression in (55) is similar to the noisy communications scenario in App. F, except for the fact that the noise has reducing variance (due to the zoom in sequence).

Following the prior analysis,

$$
\left[\Delta z_{i}(t+1)-\Delta z_{i}^{p}(t+1)\right]_{k}=\sum_{r=1}^{t+1} \sum_{j=1}^{n}\left(\frac{1}{n}-\Phi_{i j}^{k}(t+1, r)\right) s(r-1)\left[\Delta_{j}(r-1)\right]_{k}
$$

Each $s(t) \Delta_{i}(t)$ is bounded and hence sub-Gaussian with variance proxy parameter $s^{2}(t)$. Thus the above difference is sub-Gaussian with parameter

$$
\begin{aligned}
\sigma^{2}\left(\left[\Delta z_{i}(t+1)-\Delta z_{i}^{p}(t+1)\right]_{k}\right) & \leq \sum_{r=1}^{t+1} s^{2}(r-1)\left\|\frac{1}{n}-\Phi_{i}^{k}(t+1, r)\right\|_{2}^{2} \\
& \leq \sum_{r=1}^{t+1} s^{2}(r-1) \sigma_{2}\left(P^{k}\right)^{2(t-r+1)}
\end{aligned}
$$

Thus with probability greater than $1-\delta$, we have

$$
\frac{1}{T} \sum_{t=1}^{T}\left\|\bar{z}(t)-z_{i}(t)\right\|_{*} \leq \frac{1}{T} \sum_{t=1}^{T}\left\|\bar{z}^{p}(t)-z_{i}^{p}(t)\right\|_{*}+\frac{1}{T} \sqrt{2 \log (2 T n d / \delta) \max _{k} \sum_{r=0}^{t} s^{2}(r) \sigma_{2}\left(P^{k}\right)^{2(t-r+1)}\|\mathbf{1}\|_{*}}
$$

As in App. F, we next note that the bounding of $\Gamma$ as in (37) can be adapted to the case of quantized communication. By again letting $g_{i}^{\prime}(t)=g_{i}(t)+\sum_{j} \Delta_{i}(t)$, we write

$$
\begin{aligned}
\Gamma & \leq \sum_{t=1}^{T} \sum_{i=1}^{n}\left\langle g_{i}^{\prime}(t), x_{i}(t)-x^{*}\right\rangle+\sum_{t=1}^{T} \sum_{i=1}^{n}\left\langle g_{i}(t)-g_{i}^{\prime}(t), x_{i}(t)-x^{*}\right\rangle \\
& \leq n\left(\sum_{t=1}^{T}\left\langle\bar{g}^{\prime}(t), y(t)-x^{*}\right\rangle-\left\langle\bar{\Delta}(t), y(t)-x^{*}\right\rangle\right) .
\end{aligned}
$$

For the first term in $57 \mathrm{a}$, we have

$$
\sum_{t=1}^{T}\left\langle\bar{g}^{\prime}(t), y(t)-x^{*}\right\rangle \leq \sum_{t=1}^{T} \alpha(t-1)\left(\|\bar{g}(t)+\bar{\Delta}(t)\|_{*}^{2}\right)+\frac{1}{\alpha(T)} \psi\left(x^{*}\right) .
$$

Considering the case of the $\ell_{2}$-norm, we get that the first term in 58 is bounded by

$$
\sum_{t=1}^{T} \alpha(t-1)\left(\|\bar{g}(t)+\bar{\Delta}(t)\|_{*}^{2}\right) \leq \sum_{t=1}^{T} \alpha(t-1)\left(L^{2}+2 s(t)\|1\|_{2} L+s^{2}(t)\right)
$$

Finally, $\left\langle\bar{\Delta}(t), y(t)-x^{*}\right\rangle$ is zero-mean and bounded as $s(t)\|1\|_{2} R$. Applying the Azuma-Hoeffding inequality, we get with probability greater than $1-\delta$

$$
\frac{1}{T} \sum_{t=1}^{T}\left\langle\bar{\Delta}(t), y(t)-x^{*}\right\rangle \leq R\|1\|_{2} \sqrt{\widehat{s^{2}}(T) \frac{\log 1 / \delta}{T}}
$$

Combining the bounds in (56), 58) and (59) yields 14 . 